\title{
ESTIMATIVA DA RESISTÊNCIA MECÂNICA À PENETRAÇÃO DE UM LATOSSOLO VERMELHO SOB CULTURA PERENE NO NORTE DO ESTADO DO PARANÁ(1)
}

\author{
Adriana Aparecida Ribon ${ }^{(2)}$ \& João Tavares Filho(3)
}

\begin{abstract}
RESUMO
Uma das propriedades físicas importantes para o manejo e estudo da qualidade física dos solos é sua resistência à penetração, uma vez que esta propriedade se apresenta relacionada a diversos atributos do solo indicadores do grau de compactação. O objetivo deste trabalho foi estimar a resistência mecânica do solo à penetração de um Latossolo Vermelho eutroférrico sob cultura perene, em três condições de umidade gravimétrica, a partir da densidade, umidade, teor de argila (Arg) e matéria orgânica do solo. A resistência mecânica do solo à penetração foi avaliada mediante o uso do penetrômetro de impacto, e os dados obtidos em impactos $\mathrm{dm}^{-1}$ foram convertidos em resistência dinâmica (MPa). No momento da determinação da resistência mecânica do solo, foram coletadas 30 amostras por profundidade: $0-0,10 ; 0,1-0,3 ; 0,3-0,5 \mathrm{~m}$, para determinação de três diferentes teores de umidade gravimétrica (Ug) $\left(\mathrm{Ug} 1<0,20 \mathrm{~kg} \mathrm{~kg}^{-1}, 0,20 \leq \mathrm{Ug} 2<0,30 \mathrm{~kg} \mathrm{~kg}^{-1} \mathrm{e}\right.$ Ug3 $>0,30 \mathrm{~kg} \mathrm{~kg}^{-1}$ ), composição granulométrica, teor de matéria orgânica (MO) e densidade do solo (Ds). Foram aplicados testes de distribuição normal para seleção das variáveis participantes dos modelos, por meio do teste W. Em seguida, foram realizadas análises de correlação simples entre as variáveis e análise de regressão múltipla por Stepwise. Para obtenção dos modelos, realizou-se uma análise com quatro variáveis independentes, representadas por: $\mathrm{X}_{1}=\mathrm{Ug}, \mathrm{X}_{2}=\mathrm{Ds}, \mathrm{X}_{3}=\mathrm{MO}, \mathrm{X}_{4}=$ Arg, e a variável dependente $(\mathrm{Y})$, caracterizada pela resistência do solo à penetração (RP). Após obtenção dos modelos finais, testaram-se os efeitos de multicolinearidade por meio do VIF (Fator de Incremento de Variância), sendo os valores de VIF menores que 10 indicativos de que o efeito da multicolineridade não influencia os resultados da regressão. Foi utilizado o nível de significância do valor $\mathrm{F}$ da variável de 15 \% de probabilidade para a inclusão e exclusão de variáveis no modelo. Os resultados permitiram concluir que as equações que melhor estimaram a resistência do solo à penetração foram: para $\mathrm{Ug}<0,20 \mathrm{~kg} \mathrm{~kg}^{-1}$ : RPE = $1,12+2,04 \mathrm{Ds}+0,17 \mathrm{MO}\left(\mathrm{R}^{2}=0,92\right)$; para $\left(0,20 \leq \mathrm{Ug}<0,30 \mathrm{~kg} \mathrm{~kg}^{-1}\right): \mathrm{RPE}=2,93-$
\end{abstract}

\footnotetext{
(1) Parte da tese de doutorado do primeiro autor (projeto financiado pela CAPEs). Recebido para publicação em outubro de 2007 e aprovado em julho de 208.

(2) Engenheiro-Agrônomo, Doutorado em Agronomia pela, Universidade Estadual de Londrina - UEL. Caixa Postal 6001, CEP 86051-990 Londrina (PR). E-mail: aaribon@yahoo.com.br

(3) Professor Associado do Departamento de Agronomia, UEL. E-mail: tavares@uel.br
} 
$17,85 \mathrm{Ug}+2,98 \mathrm{Ds}\left(\mathrm{R}^{2}=0,95\right) ;$ para $\mathrm{Ug}>0,30 \mathrm{~kg} \mathrm{~kg}^{-1}: \mathrm{RPE}=3,20-13,78 \mathrm{Ug}+4,43 \mathrm{Ds}$ $\left(R^{2}=0,97\right)$. Em todas as situações estudadas, os coeficientes de variação variaram de altos (situação UG1) a muito altos (situação UG2 e Ug3), caracterizando alta variabilidade espacial da RP; logo, a utilização dos modelos selecionados deve ser feita com cautela, pois ocorre importante dispersão de pontos estimados por meio dos modelos, em relação aos valores medidos.

Termos de indexação: compactação, sistemas de manejo, densidade do solo, umidade gravimétrica, modelo, penetrometria de impacto.

\title{
SUMMARY: ESTIMATING PENETRATION RESISTANCE OF AN OXISOL UNDER PERENNIAL CROP IN NORTHERN PARANÁ STATE
}

\begin{abstract}
Soil resistance to root penetration is an important physical characteristic for defining soil management techniques. The purpose of this study was to estimate the mechanical soil penetration resistance of an Oxisol under perennial crop, at three gravimetrical moisture contents, based on bulk density, moisture, and soil clay and organic matter content. The soil penetration resistance was evaluated by an impact penetrometer and the impact data in $\mathrm{dm}^{-1}$ were converted into dynamic resistance $(\mathrm{MPa})$. At the moment of the determination of the mechanical soil resistance, samples (30 samples for each layer $(0-0.10 ; 0.1-$ $0.3 ; 0.3-0.5 \mathrm{~m})$ ) were collected to determine three different gravimetrical moisture contents $(U g)\left(U g 1<0.20 \mathrm{~kg} \mathrm{~kg}^{-1}, 0.20 \leq U g 2<0.30 \mathrm{~kg} \mathrm{~kg}^{-1}\right.$ and $\left.U g 3>0.30 \mathrm{~kg} \mathrm{~kg}^{-1}\right)$, clay texture (Arg), organic matter (MO) and soil bulk density (Ds). Tests of normal distribution were applied to select the participant variables of the models by the $W$ test. Thereafter, analyses of simple correlation between the variable and STEPWISE multiple regression analyses were performed. To fit the models an analysis was performed with four independent variables, represented by: $X_{1}=U g, X_{2}=D s, X_{3}=M O, X_{4}=A r g$ and the dependent variable (y) was represented by the soil penetration resistance $(R P)$. After establishing the final models, the effects of multicollinearity were tested by VIF (Variance Increase Factor), where the VIF values < 10 indicate that the effect of multicollinearity does not influence the results of regression. A significance level of $15 \%$ probability of the $F$ value of the variable was used for the inclusion or exclusion of a variable from the model. Results led to the conclusion that the equations that best estimated soil penetration resistance were: for $U g<$ $0.20 \mathrm{~kg} \mathrm{~kg}^{-1}: R P E=1.12+2.04 \mathrm{Ds}+0.17 \mathrm{MO}\left(R^{2}=0.92 ; R=0.96\right)$; for $(0.20 \leq U g<$ $\left.0.30 \mathrm{~kg} \mathrm{~kg}^{-1}\right): R P E=2.93-17.85 \mathrm{Ug}+2.98 D \mathrm{~s}\left(R^{2}=0.95, R=0.97\right) ;$ for $U g>0.30 \mathrm{~kg} \mathrm{~kg}^{-1}: R P E$ $=3.20-13.78 U g+4.43 D s\left(R^{2}=0.97, R=0.98\right)$. In all studied situations the coefficients of variation $(C V)$ varied from high (situation UG1) to very high (situation UG2 and Ug3). This high spatial variability of the RP means that care must be taken in the use of the models selected since points estimated by the models are dispersed in relation to the measured values.
\end{abstract}

Index terms: compaction, management systems, bulk density, gravimetric moisture, impact penetrometry, model.

\section{INTRODUÇÃO}

Uma das propriedades físicas importantes para o manejo e estudo da qualidade física dos solos é sua resistência à penetração, uma vez que essa propriedade apresenta-se relacionada a diversos atributos do solo indicadores do grau de compactação. Devido às relações encontradas, esse tipo de estudo, juntamente com outros atributos do solo também indicadores do grau de compactação, como a densidade do solo, porosidade e conteúdo de água, em solos cultivados, é de fundamental importância para escolha do sistema de preparo mais adequado ao crescimento e desenvolvimento das culturas.

De modo geral, o manejo inadequado dos solos cultivados provoca aumento na sua resistência à penetração e densidade, bem como diminuição da porosidade e do conteúdo de água disponível às plantas; a densidade do solo apresenta relação direta com a resistência do solo à penetração (Beltrame et al., 1981; Busscher, 1990; Busscher et al., 1997), enquanto o conteúdo de água no solo influi inversamente na 
resistência (Beltrame et al.,1981; Busscher et al., 1997; Tormena et al., 1998; Correchel et al., 1999).

$\mathrm{Na}$ literatura, vários são os modelos ou funções que buscam a obtenção de indicadores da qualidade física dos solos e que incorporam os seus atributos físicos que influenciam a compactação, tanto para as regiões temperadas (Busscher, 1990; Carter, 1990; Mairinque \& Jones, 1991; Sulivan, 1992; Smith et al., 1997) quanto para solos tropicais (Assouline et al., 1997; Imhoff et al., 2000; Cunha et al., 2002; Ribon \& Tavares Filho, 2004; Leonel et al., 2007; Lima et al., 2007). Entretanto, algumas pesquisas vêm mostrando que essas relações não são tão simples assim quando avaliadas em condições de campo (Ribon, 2004; Ribon \& Tavares Filho, 2004). Prado et al. (2002) constataram, em condições de campo, correlação nãosignificativa $(r=0,45 \mathrm{~ns})$ entre a resistência do solo à penetração e a densidade do solo na camada de 0 0,4 m de um Latossolo Vermelho eutroférrico sob cultivo intensivo e após cinco anos de pousio, com umidade gravimétrica na faixa de $0,24-0,27 \mathrm{~kg} \mathrm{~kg}^{-1}$.

Por outro lado, de acordo com Dias Júnior \& Pierce (1996), o uso desses modelos ou funções promove aumento na compreensão do processo de compactação, com conseqüente minimização do problema. Entretanto, para obtenção de modelos dentro da realidade, é necessário efetuar corretamente a observação, a coleta, a organização, a interpretação dos dados e a construção do modelo (Yaalon, 1994). Para esse autor, sendo o modelo geral uma simplificação da condição real, é necessário que haja entendimento dos processos da natureza e de suas interações para evitar que ele seja inadequado.

Smith et al. (1997), trabalhando em áreas ocupadas por espécies florestais, estabeleceram modelos de regressão, mostrando que a relação entre a resistência mecânica e densidade do solo e o conteúdo de água foi influenciada pelo conteúdo de argila e $\mathrm{C}$ orgânico para solos com menos de $30 \%$ de argila e somente pelo conteúdo de argila para solos com mais de $30 \%$ de argila. Segundo Larson et al. (1980), o processo de compactação do solo é influenciado pela textura; solos com diferentes classes texturais apresentam comportamentos distintos, quanto às propriedades físicas, quando submetidos à compactação. Outro fator importante que deve ser considerado no processo de compactação dos solos é o conteúdo de matéria orgânica (Assouline et al., 1997).

Carter (1990) estabeleceu um modelo, por meio da análise Stepwise, para avaliar as relações entre as propriedades mecânicas resistência ao cisalhamento e resistência do solo à penetração, bem como a densidade do solo e macroporosidade em solos de regiões temperadas, verificando relação positiva entre o conteúdo de $\mathrm{C}$ orgânico e a variável dependente resistência ao cisalhamento, sendo esse modelo expresso por: resistência ao cisalhamento $=-13,0+$ 29,89 (densidade do solo) -0,37 (macroporosidade) -0,29 (areia fina) + 0,67 (argila) + 1,52 (carbono orgânico)
Portanto, levar em consideração o maior número possível de atributos do solo que influenciam sua qualidade física parece ser importante para obtenção de modelos que dêem respostas confiáveis quando utilizados. Percebe-se que a resistência do solo à penetração, juntamente com a densidade e a umidade do solo, são atributos importantes, que podem contribuir como indicadores da qualidade física (grau de compactação) de solos cultivados. Entretanto, os estudos que relacionam esses fatores em condições de campo e consideram a contribuição da textura e da matéria orgânica nesses modelos são escassos em solos tropicais. O objetivo deste trabalho foi estimar a resistência mecânica à penetração de um Latossolo Vermelho eutroférrico sob cultura perene, em três condições de umidade gravimétrica distintas, a partir da densidade, umidade, teor de argila e de matéria orgânica do solo.

\section{MATERIAL E MÉTODOS}

Os dados do presente trabalho foram coletados em uma área experimental localizada no campus da Universidade Estadual de Londrina (PR), com latitude entre $23^{\circ} 08^{\prime} 47$ " e $23^{\circ} 55$ ' 46 " sul e longitude entre $50^{\circ} 52$ ' 23 " e $51^{\circ} 19^{\prime} 11$ " oeste, a $603 \mathrm{~m}$ de altitude. O clima local é do tipo Cfa-subtropical úmido, segundo Köppen, com chuvas em todas as estações (pluviometria anual em torno de $1.260 \mathrm{~mm}$ ), e o solo é um Latossolo Vermelho eutroférrico (LVe) (Embrapa, 1999), sob cultura perene de citros, com teor médio de argila na camada de 0-0,6 m variando de 627,33 a $710,17 \mathrm{~g} \mathrm{~kg}^{-1}$.

A resistência mecânica do solo à penetração foi avaliada de forma aleatória, mediante o uso do penetrômetro de impacto - modelo IAA/Planalsucar; a massa de impacto de quatro quilos sofreu uma queda livre de $40 \mathrm{~cm}$ (Stolf et al., 1983), num total de 30 repetições. Os resultados obtidos em impactos $\mathrm{dm}^{-1}$ foram convertidos em resistência dinâmica $(\mathrm{MPa})$ por meio da fórmula (Stolf, 1991):

$$
\operatorname{RP}\left(\mathrm{kgf} \mathrm{cm}^{-2}\right)=5,6+6,89 \mathrm{~N}\left(\text { impactos } \mathrm{dm}^{-1}\right)
$$

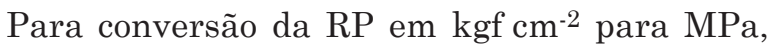
multiplicou-se o resultado obtido na equação 1 pela constante 0,0981 .

No momento da determinação da resistência mecânica do solo à penetração, foram coletadas amostras (30 amostras / profundidade) para determinação da umidade gravimétrica (Ug), teor de argila (Arg) e teor de matéria orgânica (MO), determinados de acordo com os métodos propostos pela Embrapa (1997), e da densidade do solo (Ds), determinada pelo método do anel volumétrico $\left(98 \mathrm{~cm}^{3}\right)$, em amostras indeformadas nas profundidades de $0-0,10 ; 0,1-0,3$; e $0,3-0,5 \mathrm{~m}$ (Quadro 1). 
Quadro 1. Momentos estatísticos para as variáveis analisadas nas profundidades de 0-0,1; 0,1-0,3; e 0,30,5 m, nas condições de umidade Ug1, Ug2 e Ug3, para o Latossolo Vermelho eutroférrico sob cultura perene

\begin{tabular}{|c|c|c|c|c|c|c|c|c|}
\hline \multirow{2}{*}{ Profundidade } & \multirow{2}{*}{ Variável } & \multicolumn{6}{|c|}{ Momento estatístico } & \multirow[b]{2}{*}{$\mathbf{N}$} \\
\hline & & Média & Desvio & $\mathbf{C V}$ & Moda & Máximo & Mínimo & \\
\hline \multirow[t]{2}{*}{$\mathrm{m}$} & \multicolumn{8}{|c|}{$\%$} \\
\hline & & & & Ug1 ( & $0,20 \mathrm{~kg}$ & & & \\
\hline \multirow[t]{5}{*}{$0-0,1$} & $\mathrm{RP}(\mathrm{MPa})$ & 8,03 & 1,36 & 16,96 & 6,79 & 10,97 & 6,45 & 30 \\
\hline & $\mathrm{Ug}\left(\mathrm{kg} \mathrm{kg}^{-1}\right)$ & 0,16 & 0,05 & 25,56 & 0,17 & 0,20 & 0,12 & 30 \\
\hline & $\mathrm{Ds}\left(\mathrm{Mg} \mathrm{dm}^{-3}\right)$ & 1,09 & 0,17 & 15,83 & 0,95 & 1,37 & 0,79 & 30 \\
\hline & $\mathrm{MO}\left(\mathrm{g} \mathrm{kg}^{-1}\right)$ & 9,60 & 4,65 & 48,40 & 9,38 & 16,08 & 1,34 & 30 \\
\hline & $\operatorname{Arg}\left(\mathrm{g} \mathrm{kg}^{-1}\right)$ & 644,20 & 85,20 & 12,28 & 610 & 870 & 555 & 30 \\
\hline \multirow[t]{5}{*}{$0,1-0,3$} & $\mathrm{RP}(\mathrm{MPa})$ & 6,38 & 1,83 & 28,68 & 6,79 & 11,66 & 3,32 & 30 \\
\hline & $\mathrm{Ug}\left(\mathrm{kg} \mathrm{kg}^{-1}\right)$ & 0,18 & 0,03 & 16,70 & 0,19 & 0,21 & 0,12 & 30 \\
\hline & $\mathrm{Ds}\left(\mathrm{Mg} \mathrm{dm}^{-3}\right)$ & 1,06 & 0,16 & 15,08 & 1,11 & 1,28 & 0,74 & 30 \\
\hline & $\mathrm{MO}\left(\mathrm{g} \mathrm{kg}^{-1}\right)$ & 20,51 & 6,61 & 32,23 & 20,1 & 29,49 & 4,02 & 30 \\
\hline & $\operatorname{Arg}\left(\mathrm{g} \mathrm{kg}^{-1}\right)$ & 687,80 & 94,3 & 13,71 & 490 & 865 & 490 & 30 \\
\hline \multirow[t]{6}{*}{$0,3-0,5$} & $\mathrm{RP}(\mathrm{MPa})$ & 3,95 & 1,37 & 34,76 & 4,36 & 5,17 & 0,53 & 30 \\
\hline & $\mathrm{Ug}\left(\mathrm{kg} \mathrm{kg}^{-1}\right)$ & 0,18 & 0,03 & 18,04 & 0,18 & 0,23 & 0,12 & 30 \\
\hline & $\operatorname{Ds}\left(\mathrm{Mg} \mathrm{dm}^{-3}\right)$ & 1,07 & 0,12 & 10,94 & 1,09 & 1,27 & 0,84 & 30 \\
\hline & $\mathrm{MO}\left(\mathrm{g} \mathrm{kg}^{-1}\right)$ & 12,73 & 4,27 & 33,53 & 10,72 & 22,78 & 6,70 & 30 \\
\hline & $\operatorname{Arg}\left(\mathrm{g} \mathrm{kg}^{-1}\right)$ & 699,17 & 85,19 & 12,18 & 650 & 840 & 500 & 30 \\
\hline & & \multicolumn{7}{|c|}{$\mathrm{Ug} 2\left(0,20 \leq \mathrm{Ug}<0,30 \mathrm{~kg} \mathrm{~kg}^{-1}\right)$} \\
\hline \multirow[t]{5}{*}{$0-0,1$} & $\mathrm{RP}(\mathrm{MPa})$ & 5,04 & 1,33 & 26,34 & 4,71 & 8,42 & 2,39 & 30 \\
\hline & $\mathrm{Ug}\left(\mathrm{kg} \mathrm{kg}^{-1}\right)$ & 0,25 & 0,03 & 15,14 & 0,24 & 0,32 & 0,19 & 30 \\
\hline & $\mathrm{Ds}\left(\mathrm{Mg} \mathrm{dm}^{-3}\right)$ & 1,18 & 0,19 & 16,47 & 0,98 & 1,80 & 0,93 & 30 \\
\hline & $\mathrm{MO}\left(\mathrm{g} \mathrm{kg}^{-1}\right)$ & 32,91 & 9,89 & 30,06 & 29,49 & 44,23 & 9,38 & 30 \\
\hline & $\operatorname{Arg}\left(\mathrm{g} \mathrm{kg}^{-1}\right)$ & 691,33 & 63,52 & 10,74 & 610 & 755 & 480 & 30 \\
\hline \multirow[t]{5}{*}{$0,1-0,3$} & $\mathrm{RP}(\mathrm{MPa})$ & 4,99 & 1,56 & 31,27 & 7,49 & 7,49 & 3,18 & 30 \\
\hline & $\mathrm{Ug}\left(\mathrm{kg} \mathrm{kg}^{-1}\right)$ & 0,25 & 0,03 & 10,35 & 0,24 & 0,32 & 0,22 & 30 \\
\hline & $\mathrm{Ds}\left(\mathrm{Mg} \mathrm{dm}^{-3}\right)$ & 1,21 & 0,21 & 17,45 & 1,15 & 1,70 & 0,81 & 30 \\
\hline & $\mathrm{MO}\left(\mathrm{g} \mathrm{kg}^{-1}\right)$ & 16,36 & 7,20 & 44,03 & 17,42 & 30,83 & 2,68 & 30 \\
\hline & $\operatorname{Arg}\left(\mathrm{g} \mathrm{kg}^{-1}\right)$ & 693,97 & 77,97 & 11,24 & 650 & 840 & 480 & 30 \\
\hline \multirow[t]{6}{*}{$0,3-0,5$} & $\mathrm{RP}(\mathrm{MPa})$ & 4,90 & 1,37 & 33,42 & 6,45 & 7,49 & 2,45 & 30 \\
\hline & $\mathrm{Ug}\left(\mathrm{kg} \mathrm{kg}^{-1}\right)$ & 0,26 & 0,03 & 12,50 & 0,25 & 0,31 & 0,20 & 30 \\
\hline & $\mathrm{Ds}\left(\mathrm{Mg} \mathrm{dm}^{-3}\right)$ & 1,20 & 0,18 & 15,33 & 1,19 & 1,50 & 0,88 & 30 \\
\hline & $\mathrm{MO}\left(\mathrm{g} \mathrm{kg}^{-1}\right)$ & 10,27 & 4,84 & 47,13 & 10,72 & 20,10 & 1,34 & 30 \\
\hline & $\operatorname{Arg}\left(\mathrm{g} \mathrm{kg}^{-1}\right)$ & 710,17 & 83,61 & 11,77 & 700 & 700 & 500 & 30 \\
\hline & & \multicolumn{7}{|c|}{ Ug3 $\left(\mathrm{Ug}>0,30 \mathrm{~kg} \mathrm{~kg}^{-1}\right)$} \\
\hline \multirow[t]{5}{*}{$0-0,1$} & $\mathrm{RP}(\mathrm{MPa})$ & 2,76 & 0,75 & 27,26 & 1,92 & 4,36 & 1,72 & 30 \\
\hline & $\mathrm{Ug}\left(\mathrm{kg} \mathrm{kg}^{-1}\right)$ & 0,37 & 0,12 & 31,02 & 0,25 & 0,61 & 0,21 & 30 \\
\hline & $\mathrm{Ds}\left(\mathrm{Mg} \mathrm{dm}^{-3}\right)$ & 1,06 & 0,23 & 21,87 & 0,84 & 1,87 & 0,72 & 30 \\
\hline & $\mathrm{MO}\left(\mathrm{g} \mathrm{kg}^{-1}\right)$ & 32,91 & 9,89 & 30,05 & 29,49 & 44,23 & 9,38 & 30 \\
\hline & $\operatorname{Arg}\left(\mathrm{g} \mathrm{kg}^{-1}\right)$ & 710,02 & 83,61 & 11,77 & 700 & 865 & 500 & 30 \\
\hline \multirow[t]{5}{*}{$0,1-0,3$} & $\mathrm{RP}(\mathrm{MPa})$ & 3,47 & 0,66 & 19,13 & 4,36 & 4,71 & 1,96 & 30 \\
\hline & $\mathrm{Ug}\left(\mathrm{kg} \mathrm{kg}^{-1}\right)$ & 0,39 & 0,11 & 26,78 & 0,28 & 0,55 & 0,25 & 30 \\
\hline & $\mathrm{Ds}\left(\mathrm{Mg} \mathrm{dm}^{-3}\right)$ & 1,07 & 0,16 & 15,03 & 1,12 & 1,32 & 0,72 & 30 \\
\hline & $\mathrm{MO}\left(\mathrm{g} \mathrm{kg}^{-1}\right)$ & 17,24 & 8,58 & 49,74 & 17,42 & 42,89 & 2,68 & 30 \\
\hline & $\operatorname{Arg}\left(\mathrm{g} \mathrm{kg}^{-1}\right)$ & 627,33 & 73,12 & 11,66 & 630 & 725 & 420 & 30 \\
\hline \multirow[t]{5}{*}{$0,3-0,5$} & $\mathrm{RP}(\mathrm{MPa})$ & 3,99 & 1,31 & 32,76 & 3,32 & 6,79 & 2,31 & 30 \\
\hline & $\mathrm{Ug}\left(\mathrm{kg} \mathrm{kg}^{-1}\right)$ & 0,40 & 0,11 & 27,84 & 0,29 & 0,58 & 0,26 & 30 \\
\hline & $\mathrm{Ds}\left(\mathrm{Mg} \mathrm{dm}^{-3}\right)$ & 1,00 & 0,16 & 16,07 & 0,76 & 1,25 & 0,76 & 30 \\
\hline & $\mathrm{MO}\left(\mathrm{g} \mathrm{kg}^{-1}\right)$ & 10,27 & 4,84 & 47,13 & 10,72 & 20,10 & 1,34 & 30 \\
\hline & $\operatorname{Arg}\left(\mathrm{g} \mathrm{kg}^{-1}\right)$ & 693,33 & 85,5 & 12,33 & 630 & 870 & 470 & 30 \\
\hline
\end{tabular}

RP: resistência mecânica do solo à penetração; Ug: umidade gravimétrica; Ds: densidade do solo; MO: matéria orgânica; Arg.: teor de argila. 
A coleta dessas amostras foi realizada sempre em uma mesma área, isolada durante o período de realização do trabalho, em três condições de umidade gravimétrica distintas ( $\mathrm{Ug} 1$ - $\left(\mathrm{Ug}<0,20 \mathrm{~kg} \mathrm{~kg}^{-1}\right)$, Ug2 - $\left(0,20 \leq \mathrm{Ug}<0,30 \mathrm{~kg} \mathrm{~kg}^{-1}\right)$ e Ug3 - $\left.\left(\mathrm{Ug}>0,30 \mathrm{~kg} \mathrm{~kg}^{-1}\right)\right)$, obtidas em campo após acompanhamento da umidade no perfil de solo (0-60 cm), com coletas diárias, e sempre no mesmo horário (entre 9 e 10 h), de amostras com trado holandês. Esse acompanhamento de umidade no perfil do solo ocorreu no mês de novembro de 2006 (período chuvoso na região), e a opção por trabalhar com as umidades Ug1, Ug2 e Ug3, apresentadas anteriormente, deveu-se ao fato de esses solos argilosos tenderem a apresentar umidades ótimas de compactação aproximadamente entre 0,27 e 0,30 kg kg-1 (Dias Júnior \& Miranda, 2000), provavelmente por apresentarem grande capacidade de adsorção de água (Ekwue \& Stone, 1997).

Foram aplicados testes de distribuição normal para seleção das variáveis participantes dos modelos por meio do teste W (Shapiro \& Wilk, 1965), sendo excluídas aquelas que não apresentaram distribuição normal dos valores. Em seguida, foram realizadas análises de correlação simples entre as variáveis e análise de regressão múltipla por Stepwise, utilizando o procedimento PROC REG do sistema SAS (1991) para modelar o efeito das propriedades nessas condições.

Para obtenção dos modelos, realizou-se uma análise com quatro variáveis independentes, representadas por: $\mathrm{X}_{1}$. umidade gravimétrica $(\mathrm{Ug}), \mathrm{X}_{2}$ - densidade do solo (Ds), $\mathrm{X}_{3}$ - matéria orgânica (MO) e $\mathrm{X}_{4}$ - argila (Arg), e a variável dependente $(\mathrm{Y})$, caracterizada pela resistência do solo à penetração $(\mathrm{RP})$. Após obtenção dos modelos finais, testaram-se os efeitos de multicolinearidade por meio do VIF (Fator de
Incremento de Variância), sendo os valores de VIF menores que 10 indicativos de que o efeito da multicolineridade não está apresentando influência dos resultados da regressão (Neter et al., 1989). No presente trabalho, todas as variáveis participantes dos modelos finais apresentaram valores de VIF menores que 10.

Foi utilizado o nível de significância do valor $\mathrm{F}$ da variável de $15 \%$ para a inclusão e exclusão de variáveis no modelo, de acordo com Dias et al. (1999). Uma vez estabelecidos os modelos para estimativa da resistência do solo à penetração nas diferentes umidades e profundidades para um mesmo manejo e solo, as médias obtidas em campo e determinadas a partir desses modelos foram comparadas entre si pelo teste de Tukey a 5 \%. Em seguida, fez-se a seleção de um modelo por meio do coeficiente de determinação $\left(\mathrm{R}^{2}\right)$ (Draper \& Smith, 1981).

\section{RESULTADOS E DISCUSSÃO}

Pode-se observar alto coeficiente de variação (Gomes, 1984) para a resistência do solo à penetração e matéria orgânica, devido à grande amplitude dos valores para esses dois atributos, e baixa amplitude de variação para as outras variáveis (Quadro 1).

Foram desenvolvidas três equações paras as diferentes umidades gravimétricas e profundidades para um mesmo manejo e solo; as condições estabelecidas mostraram algumas diferenças quanto às variáveis selecionadas que melhor estimaram a variação da resistência do solo à penetração (Quadro 2), como visto a seguir:

- Para a condição Ug1 (Ug média do solo < 0,20 kg kg-1), observa-se que não houve diferença significativa a

Quadro 2. Modelos selecionados para a estimativa da resistência do solo à penetração (RPE) por meio do coeficiente de determinação $\left(R^{2}\right)$, considerando a umidade gravimétrica (Ug), densidade do solo (Ds) e matéria orgânica (MO) do Latossolo Vermelho eutroférrico sob cultura perene, em três condições de umidade gravimétrica (Ug1, Ug2 e Ug3) nas profundidades

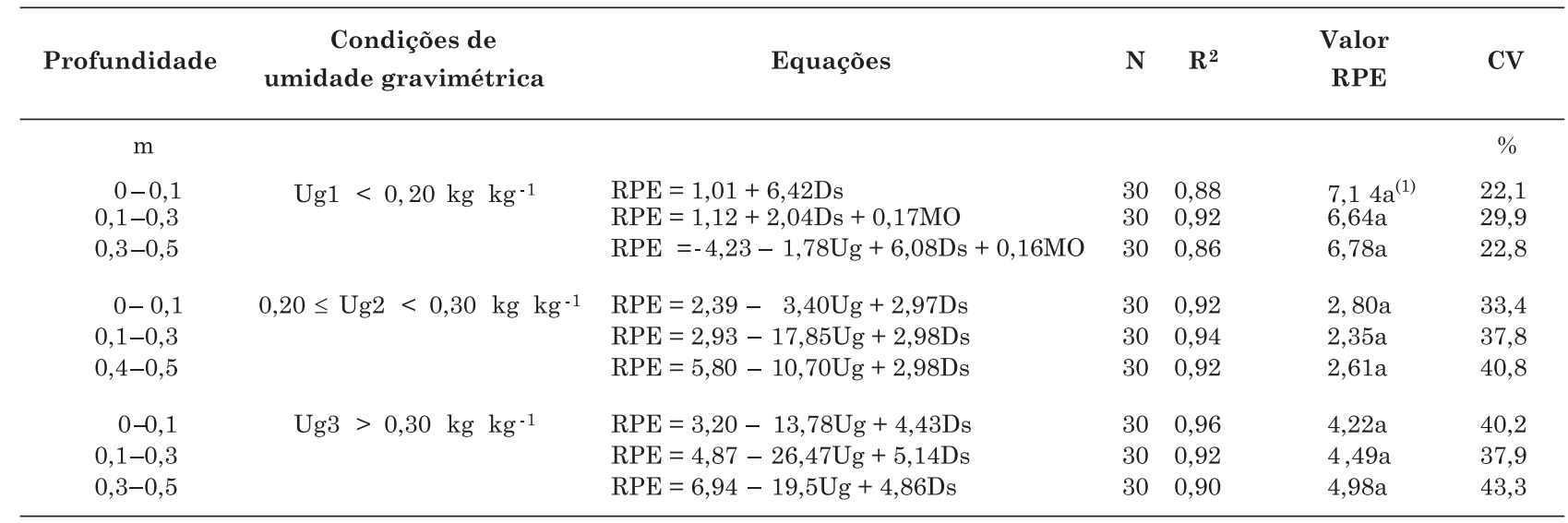

Médias seguidas de mesma letra minúscula na coluna não diferem significativamente entre si pelo teste de Tukey a 5 \%; CV: coeficiente de variação. 
$5 \%$ pelo teste de Tukey entre as RPEs, e os coeficientes de determinação apresentaram valores próximos para as três profundidades estudadas: $R^{2}$ variou de 0,86 a 0,96 , porém, para a profundidade de $0-0,1 \mathrm{~m}$, a variável densidade do solo (Ds) foi a que mais contribuiu para estimativa da resistência do solo à penetração estimada (RPE); neste caso, o modelo estabelecido apresentou $R^{2}=0,88$, demonstrando que no grupo de amostras analisado a variável Ds explicou $88 \%$ da variância da RPE. Na profundidade de 0,1-0,3 m, além da variável Ds, a variável matéria orgânica (MO) também contribuiu para a estimativa da RPE; o modelo estabelecido apresentou $\mathrm{R}^{2}=0,92$, demonstrando que no grupo de amostras analisado essas variáveis explicaram $92 \%$ da variância da RPE. No caso da profundidade de $0,3-0,5 \mathrm{~m}$, as variáveis que mais contribuíram para a estimativa da RPE foram a umidade gravimétrica do solo (Ug), a Ds e a MO, sendo o modelo estabelecido com um $R^{2}=0,86$, demonstrando que, no grupo de amostras analisado, essas variáveis explicaram 86 \% da variância da RPE.

Verifica-se que ocorre variação nos fatores que mais influenciam a RPE, segundo a profundidade considerada para essa condição de umidade (Ug1), ou seja, a variável Ds aparece nos modelos das três profundidades estudadas, enquanto a variável MO não aparece somente na profundidade de $0,0-0,1 \mathrm{~m}$ e a variável $\mathrm{Ug}$ aparece apenas na profundidade de 0,3-0,5 $\mathrm{m}$. Portanto, das três equações elaboradas, a escolhida para estimar a resistência do solo à penetração, para essa condição de estudo, foi aquela que apresentou o maior $R^{2}$, ou seja: $\mathrm{RPE}=1,12+2,04 \mathrm{Ds}+0,17 \mathrm{MO}$.

- Para as condições Ug2 (Quadro 2: Ug média entre 0,20 e $0,30 \mathrm{~kg} \mathrm{~kg}^{-1}$ ) e Ug3 (Ug média > 0,30 kg kg-1), a resistência à penetração pode ser estimada, independentemente da profundidade, pela expressão $\mathrm{RPE}=\mathrm{a}-\mathrm{bUg}+\mathrm{CDs}$; para as duas situações, não houve diferença significativa a $5 \%$ pelo teste de Tukey entre as RPEs e, no caso da umidade U2, o maior valor de $R^{2}=0,94$ foi encontrado na profundidade de 0,1-0,3 $\mathrm{m}$, demonstrando que no grupo de amostras analisado para essa condição essas variáveis explicaram em $94 \%$ a variância da RPE, enquanto, no caso da umidade do solo U3, o $\mathrm{R}^{2}=0,96$ foi encontrado na profundidade de $0-0,1 \mathrm{~m}$, demonstrando que no grupo de amostras analisado para essa condição essas variáveis explicaram $94 \%$ da variância da RPE.

Outro aspecto do observado (Quadro 2) são os coeficientes de variação (CV), que variam de altos (situação UG1) a muito altos (situação UG2 e Ug3), indicando que seria melhor trabalhar com número maior que 30 repetições $(\mathrm{N})$, uma vez que no campo a RP é bastante variável (alta variabilidade espacial), sendo muito influenciada pelas condições do manejo do solo e à intensidade de tráfego de máquinas, e é dependente da umidade do solo (Silva et al., 2004).
A partir desses resultados, verifica-se que a densidade do solo aparece com importante contribuição em todas as condições analisadas, indicando relação direta com a resistência do solo à penetração, o que está de acordo com vários trabalhos (Beltrame et al., 1981; Busscher, 1990; Busscher et al., 1997). A umidade do solo somente se tornou presente em todas as condições analisadas quando se encontrava acima de $0,20 \mathrm{~kg} \mathrm{~kg}^{-1}$, além de mostrar sua influência inversamente proporcional à resistência, como relatado por alguns autores (Beltrame et al.,1981; Busscher et al., 1997; Tormena et al., 1998; Correchel et al., 1999).

Em nenhuma situação a variável teor de argila (Arg) contribuiu para estimativa da resistência do solo à penetração, provavelmente por existir pouca variação desse teor na área analisada. Por outro lado, Ribon \& Tavares Filho (2004), trabalhando em Latossolo Vermelho-Amarelo sob pastagem, encontraram contribuição do teor de argila no modelo para estimativa da resistência do solo. Essa diferença nos resultados encontrados para esses dois tipos de Latossolo pode ser devido ao fato de freqüentemente o LV apresentar textura argilosa/muito argilosa (com microestrutura bem desenvolvida), enquanto o LVA freqüentemente apresenta textura média. Isso indica a importância de se considerar que os modelos apresentados somente devem ser indicados para solos com textura próxima à do solo estudado neste trabalho, principalmente no caso do modelo baseado somente na densidade do solo, o qual, se utilizado em solos com textura arenosa, portanto com alta densidade resultante da mineralogia do solo, poderia resultar em altos valores estimados para a resistência do solo, o que pode não ser verdadeiro para esse tipo de solo.

Em relação ao teor de matéria orgânica, observase que ele influenciou os modelos somente na condição de umidade do solo Ug1 (Ugg < 0,20 kg kg-1), a partir de $0,10 \mathrm{~m}$ de profundidade. Relação direta entre a resistência à penetração e o teor de matéria orgânica também foi observada em trabalhos conduzidos por Carter (1990) e Ribon \& Tavares Filho (2004). Provavelmente, essa relação direta obtida entre a MO e a resistência mecânica pode ser atribuída ao fato de a MO possuir a propriedade de cimentação das partículas, facilitando a formação de estruturas em blocos, o que leva ao aumento na resistência mecânica e densidade do solo. Isso indica, portanto, pelos resultados obtidos, a necessidade de mais informações a respeito da influência da MO na compactação dos solos tropicais, pois esta é uma propriedade importante para estimativa da qualidade física dos solos. De acordo com Elkwude \& Stone (1995), a influência da MO na compactação dos solos depende não apenas do teor da MO, mas também do tipo de material orgânico presente. Entretanto, estudos mais detalhados seriam necessários para atribuir esse fato ao tipo ou estádio de decomposição da MO nesse solo.

Por outro lado, a relação entre a $\mathrm{MO}$ e a resistência do solo, obtida neste trabalho, não está totalmente em 
acordo com a encontrada por Ekwue \& Stone (1995) em condições laboratoriais, pois, de acordo com eles, a MO (teores entre 17 e $27 \mathrm{~g} \mathrm{~kg}^{-1}$ ) reduziu a resistência do solo em baixos conteúdos de umidade e aumentou em altos níveis de umidade, o que não ocorreu nesse caso, pois a relação direta entre a MO e a resistência do solo foi somente para a condição de umidade do solo U1.

A umidade gravimétrica foi regularmente selecionada nos modelos quando o solo apresentou $\mathrm{Ug}>0,20 \mathrm{~kg} \mathrm{~kg}^{-1}$. Os resultados para o caso da umidade U1 estão em acordo com os obtidos por Ribon \& Tavares Filho (2004), os quais, trabalhando com Latossolo Vermelho-Amarelo sob pastagem, também verificaram a não-inclusão da umidade gravimétrica no modelo final obtido. Esses autores atribuíram a falta de relação encontrada entre a umidade gravimétrica e outras propriedades físicas do solo avaliadas à pequena amplitude de umidade encontrada quando da execução do trabalho $\left(0,21-0,25 \mathrm{~kg} \mathrm{~kg}^{-1}\right)$. Também Ribon (2000), trabalhando com uma pequena faixa de variação de umidade gravimétrica em solo sob cultura perene a campo, por meio da análise de covariância para correção das médias de resistência do solo à penetração pela umidade gravimétrica, observou que os valores das médias corrigidas foram próximos aos das médias obtidas, mostrando pouca influência da umidade gravimétrica nos valores de penetrometria obtidos.

A densidade do solo foi a variável selecionada que teve a maior participação em todas as condições de umidade estabelecidas. Esse resultado está em desacordo com Prado et al. (2002), que encontraram correlação não-significativa entre a resistência e a densidade do solo trabalhando em Latossolo Vermelho eutroférrico a campo, ao verificarem alterações na resistência à penetração e densidade do solo durante cultivo intensivo e após cinco anos de pousio na camada de 0-0,4 m, porém numa faixa de umidade considerada na capacidade de campo $\left(0,24-0,27 \mathrm{~kg} \mathrm{~kg}^{-1}\right)$; também está em desacordo com a afirmação de Larson (1964), que atribui a correlação não-significativa entre a resistência à penetração e a densidade do solo ao fato de esta não ser considerada uma medida direta de resistência.

Observa-se que não existe diferença significativa a $5 \%$ pelo teste de Tukey entre as médias de resistência RPC e RPE (Quadro 3).

Em relação aos valores estimados pelas equações apresentadas (Quadro 3), percebe-se, para as três situações estudadas (Figura 1), que os pontos estimados não estão próximos dos valores reais, como pode ser observado pela dispersão dos pontos em torno da reta 1:1, a qual indica o que seria um ajuste perfeito, ou seja, quanto mais próximo dela, maior a exatidão da estimativa (Wösten et al., 1990; Zhuang et al., 2001). Além disso, o intervalo de confiança (95\%) é expressivo em todas as três situações estudadas, portanto, a utilização dos modelos selecionados deve ser feita com cautela.

Mesmo assim, pode-se considerar que os modelos propostos neste trabalho, utilizando análise de regressão múltipla por Stepwise, possibilitaram bons ajustes, tendo em vista os valores de $\mathrm{R}^{2}$ encontrados, principalmente por se tratar de uma propriedade do solo que, normalmente, apresenta grande variabilidade. $\mathrm{O}$ uso de modelagens estatísticas pode proporcionar grandes evoluções em termos de exatidão, e são válidos estudos dessa natureza (Cornelis et al., 2001), uma vez que possibilita melhor compreensão de quais atributos devem ser considerados na estimativa da resistência à penetração desses solos argilosos, sob cultura perene.

Quadro 3. Relação entre os valores de resistência à penetração determinados a campo (RPC) e estimados (RPE) em função da umidade gravimétrica (Ug), densidade do solo (Ds) e matéria orgânica (MO) do Latossolo Vermelho eutroférrico sob cultura perene

\begin{tabular}{|c|c|c|c|c|c|}
\hline \multirow{2}{*}{ Umidade gravimétrica } & \multirow{2}{*}{$\mathbf{N}$} & \multicolumn{2}{|c|}{ Resistência à Penetração (MPa) } & \multicolumn{2}{|c|}{ CV } \\
\hline & & RPC & RPE & RPC & RPE \\
\hline $\mathrm{kg} \mathrm{kg}^{-1}$ & & & & $\longrightarrow$ & - \\
\hline $\mathrm{Ug} 1<0,20$ & 30 & $6,49 a$ & $\begin{array}{c}\mathrm{RPE}=1,12+2,04 \mathrm{Ds}+0,17 \mathrm{MO} \\
6,64 \mathrm{a}\end{array}$ & 26,6 & 29,9 \\
\hline $0,20 \leq \mathrm{Ug} 2<0,30$ & 30 & $2,27 \mathrm{a}$ & $\begin{array}{c}\mathrm{RPE}=2,93-17,85 \mathrm{Ug}+2,98 \mathrm{Ds} \\
2,35 \mathrm{a}\end{array}$ & 29,2 & 33,4 \\
\hline $\mathrm{Ug} 3>0,30$ & 30 & $4,15 \mathrm{a}$ & $\begin{array}{c}\mathrm{RPE}=3,20-13,78 \mathrm{Ug}+4,43 \mathrm{Ds} \\
4,22 \mathrm{a}\end{array}$ & 37,4 & 40,2 \\
\hline
\end{tabular}

Médias seguidas de mesma letra minúscula na linha não diferem significativamente entre si pelo teste de Tukey a 5 \%; CV: coeficiente de variação. 

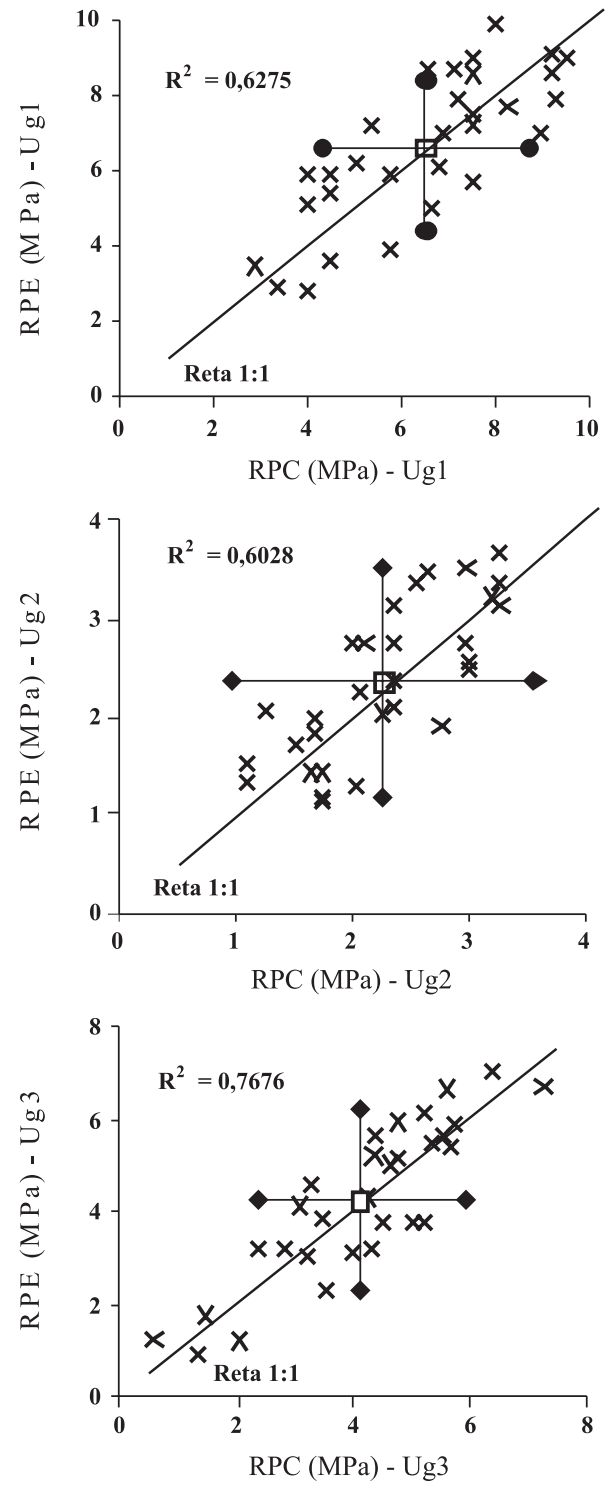

Figura 1. Representação dos valores de resistência do solo à penetração estimados (RPE) a partir das equações apresentadas no quadro 3 , em relação aos valores de resistência do solo à penetração determinados a campo (RPC) e aos intervalos de confiança (95\%) da RPE e RPC, para três umidades gravimétricas consideradas em Latossolo Vermelho sob cultura perene.

\section{CONCLUSÕES}

1. As equações que melhor estimaram a resistência do solo à penetração foram: para Umidade gravimétrica $(\mathrm{Ug} 1)<0,20 \mathrm{~kg} \mathrm{~kg}^{-1}$ : Resistência à Penetração Estimada = 1,12 + 2,04 Densidade do solo $+0,17$ teor Matéria orgânica $\left(\mathrm{R}^{2}=0,92\right)$; para $(0,20$ $\leq$ Umidade gravimétrica $\left.(\mathrm{Ug} 2)<0,30 \mathrm{~kg} \mathrm{~kg}^{-1}\right)$ : Resistência à Penetração Estimada $=2,93-17,85$ Umidade gravimétrica $+2,98$ Densidade do solo $\left(\mathrm{R}^{2}=\right.$ 0,95); para Umidade gravimétrica (Ug3) $>0,30 \mathrm{~kg} \mathrm{~kg}^{-1}$ :
Resistência à Penetração Estimada $=3,20-13,78$ Umidade gravimétrica + 4,43 Densidade do solo $\left(\mathrm{R}^{2}=\right.$ 0,97).

2. Em todas as situações estudadas, os coeficientes de variação (CV) variaram de altos (situação Ug1) a muito altos (situação Ug2 e Ug3), caracterizando alta variabilidade espacial da $R P$.

3. A utilização dos modelos selecionados deve ser feita com cautela, pois ocorre importante dispersão de pontos estimados por meio deles, em relação aos valores medidos.

\section{LITERATURA CITADA}

ASSOULINE, S.; TAVARES FILHO, J. \& TESSIER, D. Effect of compaction on soil physical properties: Experimental results and modeling. Soil Sci. Soc. Am. J., 61:391-398, 1997.

BELTRAME, L.F.S.; GONDIM, L.A.P. \& TAYLOR, J.C. Estrutura e compactação na permeabilidade de solos do Rio Grande do Sul. R. Bras. Ci. Solo, 5:145-149, 1981.

BUSSCHER, W.J. Adjustment of flat-tipped penetrometer resistance data to a common water content. Trans. Am. Soc. Agron. Eng., 33:519-524, 1990.

BUSSCHER, W.J.; BAUER, P.J.; CAMP, C.R. \& SOJKA, R.E. Correction of cone index water content differences in a Coastal Plain soil. Soil Till. Res., 43:205-217, 1997.

CARTER, M.R. Relations of strength properties to bulk density and macroporosity in cultivated loamy sand to loam soils. Soil Till. Res., 15:257-268, 1990.

CORNELIS, W.M.; RONSYN, J.; MEIRVENNE, M.van \& HARTMANN, R. Evaluation of pedotransfer functions for predicting the soil moisture retention curve. Soil Sci. Soc. Am. J., 65:638-648, 2001.

CORRECHEL, V.; SILVA, A.P. \& TORMENA, C.A. Influência da posição relativa à linha de cultivo sobre a densidade do solo em dois sistemas de manejo do solo. R. Bras. Ci. Solo, 23:165-173, 1999 .

CUNHA, J.P.A.R.; VIEIRA, L.B. \& MAGALHÃES, C.C. Resistência mecânica do solo à penetração sob diferentes densidades e teores de água. Eng. Agric., 10:1-7, 2002.

DIAS JÚNIOR, M.S \& PIERCE, F.J. Revisão de literatura: O processo de compactação do solo e sua modelagem. R. Bras. Ci. Solo, 20:175-182, 1996.

DIAS JÚNIOR, M.S. \& MIRANDA, E.E.V. Comportamento da curva de compactação de cinco solos da região de Lavras (MG). Ci. Agrotec., 24:337-346, 2000.

DIAS, F.L.F.; MAZZA, J.A.; MATSUOKA, S.; PERECIN, D. \& MAULE, R.F. Relação entre produtividade, clima, solos e variedades de cana-de-açúcar na região noroeste do Estado de São Paulo. R. Bras. Ci. Solo, 23:627-634, 1999.

DRAPER, N.R. \& SMITH, H. Applied regression analysis. 2.ed. New York, John \& Wiley, 1981. 407p. 
EKWUE, E.J. \& STONE, R.J. Density-moisture relations of some Trinidadian soils incorporated with sewage sludge. Trans. Am. Soc. Agron. Eng., 40:317-323, 1997.

EKWUE, E.J. \& STONE, R.J. Organic matter effects on strength properties of compacted agricultural soils. Trans. Am. Soc. Agron. Eng., 38:357-365, 1995.

EMPRESA BRASILEIRA DE PESQUISA AGROPECUÁRIA EMBRAPA. Centro Nacional de Pesquisa de Solos. Sistema brasileiro de classificação de solos. Brasília, 1999. $412 \mathrm{p}$.

EMPRESA BRASILEIRA DE PESQUISA AGROPECUÁRIA EMBRAPA. Serviço Nacional de Levantamento e Conservação de Solos. Manual de métodos de análise de solo. 2.ed. Rio de Janeiro, 1997. 212p.

GOMES, F.P. A estatística moderna na pesquisa agropecuária. Piracicaba, Associação Brasileira para Pesquisa da Potassa e do Fosfato, 1984. 160p.

IMHOFF, S.; SILVA, A.P. \& TORMENA, C.A. Aplicações da curva de resistência no controle da qualidade física de um solo sob pastagem. Pesq. Agropec. Bras., 35:1493-1500, 2000 .

LARSON, W.E. Soil parameters for evaluating tillage needs and operations. Soil Sci. Soc. Am. Proc., 28:118-122, 1964.

LARSON, W.E.; GUPTA, S.C. \& USECHE, R.A. Compression of agricultural soils from eight soil orders. Soil Sci. Soc. Am. J., 44:450-457, 1980.

LEONEL, C.L.; CENTURION, M.A.P.C.; CENTURION, J.F.; BEUTLER, A.N. \& FREDDI, O.S. Relação da compactação do solo com a cultura do amendoim. Biosci. J., 23:7081, 2007.

LIMA, C.L.R.; REICHERT, J.M.; REINERT, D.J.; SUZUKI, L.E.A.S. \& DALBIANCO, L. Densidade crítica ao crescimento de plantas considerando água disponível e resistência à penetração de um Argissolo Vermelho distrófico arênico. Ci. Rural, 37:1166-1169, 2007.

MAIRINQUE, L.A. \& JONES, C.A. Bulk density of soil in relation to soil physical and chemical properties. Soil Sci. Soc. Am. J., 55:476-480, 1991.

NETER, J.; WASSERMAN, W. \& KUTNER, M.H. Applied linear regression models. 2.ed. Homewood, R. Irwin, 1989. $245 \mathrm{p}$.

PRADO, R.M.; ROQUE, C.G. \& SOUZA, Z.M. Sistemas de manejo e resistência do solo à penetração e densidade do solo de um Latossolo Vermelho eutroférrico em cultivo intensivo e pousio. Pesq. Agropec. Bras., 37:1795-1801, 2002.
RIBON, A.A. Estudo da resistência a penetração em Latossolos. Londrina, Universidade Estadual de Londrina, 2004. 113p. (Tese de Doutorado)

RIBON, A.A. Propriedades físicas de Latossolo e Podzólico cultivados com seringueira (Hevea brasiliensis) submetidos a práticas de manejos no Planalto Ocidental Paulista. Jaboticabal, Universidade Estadual de São Paulo, 2000. 130p. (Tese de Mestrado)

RIBON, A.A. \& TAVARES FILHO, J. Model propositions for the estimation of the physical quality of a Yellow Red Latosol (Oxisol) under pasture. Braz. Arch. Biol. Technol., 47:25-31, 2004.

SAS Institute. SAS/STAT procedure guide for personal computers. Version 5.ed. Cary, 1991.

SHAPIRO, S.S. \& WILK, M.B. An analysis of variance test for normality (complete samples). Biometrika, 52:591-611, 1965 .

SILVA, V.R.; REICHERT, J.M. \& REINERT, D.J. Variabilidade espacial da resistência do solo à penetração em plantio direto. Ci. Rural, 34:399-406, 2004.

SMITH, C.W.; JOHNSTON, M.A. \& LORENTZ, S. The effect of soil compaction and soil physical properties on the mechanical resistance of South African forest soils. Geoderma, 78:93-111, 1997.

STOLF, R. Teoria e teste experimental de fórmulas de transformação dos dados de penetrômetro de impacto em resistência do solo. R. Bras.Ci. Solo, 15:249-252, 1991.

STOLF, R.; FERNANDES, J. \& FURLANI NETO, V.L. Penetrômetro de impacto modelo IAA/Planalsucar-Stolf: Recomendação para seu uso. STAB, 1:18-23, 1983.

SULLIVAN, M.F.O. Uniaxial compaction effects on physical properties in relation to solitype and cultivation. Soil Till. Res., 24:257-269, 1992.

TORMENA, C.A.; SILVA, A.P. \& LIBARDI, P.L. Caracterização e avaliação do intervalo hídrico ótimo de um Latossolo Roxo. R. Bras. Ci. Solo, 22:573-581, 1998.

WÖSTEN, J.H.M.; SCHUREN, C.H.J.E.; BOUMA, J. \& STEIN, A. Functional sensitivity analysis of four methods to generate soil hydraulic functions. Soil Sci. Soc. Am. J., 55:832-836, 1990.

YAALON, D.H. On models, modeling, and process understanding. Soil Sci. Soc. Am. J., 58:1276, 1994.

ZHUANG, J.; JIN, Y. \& MIYAZAKI, T. Estimating water retention characteristic from soil particle-size distribution using a non-similar media concept. Soil Sci., 166:308-321, 2001. 